\title{
Metal-Free, Regioselective Diyne Polycyclotrimerization: Synthesis, Photoluminescence, Solvatochromism, and Two-Photon Absorption of a Triphenylamine-Containing Hyperbranched Poly(aroylarylene)
}

Anjun Qin, ${ }^{\dagger}$ Jacky W. Y. Lam, ${ }^{\dagger}$ Hongchen Dong, Weixin Lu, ${ }^{\ddagger}$ Cathy K. W. Jim, ${ }^{\dagger}$ Yongqiang Dong, ${ }^{\dagger, \S}$

Matthias Häußler, ${ }^{\dagger}$ Herman H. Y. Sung, ${ }^{\dagger}$ Ian D. Williams, ${ }^{\dagger}$ George K. L. Wong, ${ }^{\ddagger}$ and Ben Zhong Tang $*,+, \S$

Department of Chemistry and Department of Physics, The Hong Kong University of Science \& Technology (HKUST), Clear Water Bay, Kowloon, Hong Kong, China, and Department of Polymer Science and Engineering, Zhejiang University, Hangzhou 310027, China

Figure S1. ORTEP drawing of $N, N$-bis(4-ethynylcarbonylphenylene)aniline (1)....................S2

Table S1-S6. Single crystal analysis data for $N, N$-bis(4-ethynylcarbonyl-phenylene)aniline (1)....S2-S8

Figure S2. ORTEP drawing of $N$-(4-ethynylcarbonylphenylene)diphenylamine (4)...............S10

Table S7-S12. Single crystal data for $N$-(4-ethynylcarbonylphenylene)diphenylamine (4)......S10-S16 


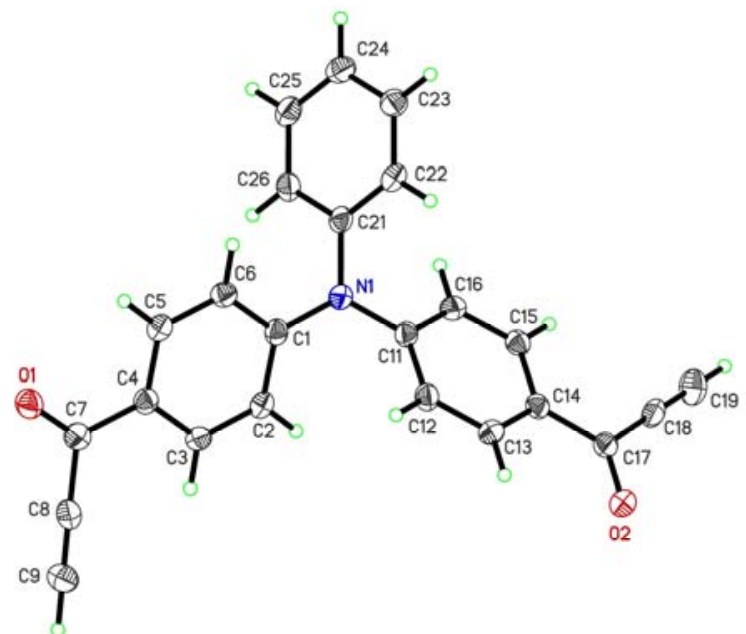

Figure S1. ORTEP drawing of $N, N$-bis(4-ethynylcarbonylphenylene)aniline (1).

Table S1. Crystal data and structure refinement for $\mathbf{1 .}$

Empirical formula

Formula weight

Temperature

Wavelength

Crystal system

Space group

Unit cell dimensions

Volume

$\mathrm{Z}$

Density (calculated)

Absorption coefficient

$\mathrm{F}(000)$

Crystal size

Theta range for data collection

Index ranges

Reflections collected

Independent reflections

Completeness to theta $=25.00^{\circ}$

Absorption correction

Max. and min. transmission

Refinement method

Data / restraints / parameters

Goodness-of-fit on $\mathrm{F}^{2}$

Final R indices [I $>2 \operatorname{sigma(I)]~}$

$\mathrm{R}$ indices (all data)

Largest diff. peak and hole
$\mathrm{C}_{24} \mathrm{H}_{15} \mathrm{NO}_{2}$

349.37

$100(2) \mathrm{K}$

$0.71073 \AA$

Monoclinic

P2(1)/n

$\mathrm{a}=10.3535(8) \AA \quad \alpha=90^{\circ}$.

$\mathrm{b}=10.2064(8) \AA \quad \beta=94.573(2)^{\circ}$.

$\mathrm{c}=17.3231(14) \AA \quad \gamma=90^{\circ}$.

1824.7(2) $\AA^{3}$

4

$1.272 \mathrm{mg} / \mathrm{m}^{3}$

$0.081 \mathrm{~mm}^{-1}$

728

$0.40 \times 0.30 \times 0.06 \mathrm{~mm}^{3}$

2.22 to $26.00^{\circ}$.

$-10<=\mathrm{h}<=12,-12<=\mathrm{k}<=12,-19<=\mathrm{l}<=21$

11708

$3542[\mathrm{R}(\mathrm{int})=0.0256]$

$99.0 \%$

Semi-empirical from equivalents

1.00 and 0.97

Full-matrix least-squares on $\mathrm{F}^{2}$

3542 / 0 / 244

1.047

$\mathrm{R} 1=0.0377, \mathrm{wR} 2=0.0929$

$\mathrm{R} 1=0.0596, \mathrm{wR} 2=0.1002$

0.239 and -0.175 e. $\AA^{-3}$ 
Table S2. Atomic coordinates $\left(\times 10^{4}\right)$ and equivalent isotropic displacement parameters $\left(\AA^{2} \times 10^{3}\right)$ for $\mathbf{1}$. $\mathrm{U}(\mathrm{eq})$ is defined as one third of the trace of the orthogonalized $\mathrm{Uij}$ tensor.

\begin{tabular}{|c|c|c|c|c|}
\hline & $\mathrm{x}$ & $\mathrm{y}$ & $\mathrm{Z}$ & $\mathrm{U}(\mathrm{eq})$ \\
\hline $\mathrm{N}(1)$ & $4604(1)$ & $1747(1)$ & 1992(1) & $24(1)$ \\
\hline $\mathrm{O}(1)$ & $3156(1)$ & $4253(1)$ & $-1367(1)$ & $31(1)$ \\
\hline $\mathrm{O}(2)$ & 6691(1) & $5056(1)$ & 4985(1) & $31(1)$ \\
\hline $\mathrm{C}(1)$ & $4230(1)$ & 2495(1) & 1332(1) & $22(1)$ \\
\hline$C(2)$ & $3712(1)$ & $3756(1)$ & 1388(1) & $25(1)$ \\
\hline $\mathrm{C}(3)$ & $3362(1)$ & $4471(1)$ & $733(1)$ & $24(1)$ \\
\hline$C(4)$ & $3476(1)$ & $3950(1)$ & $-5(1)$ & $22(1)$ \\
\hline$C(5)$ & 3963(1) & $2684(1)$ & $-58(1)$ & $23(1)$ \\
\hline$C(6)$ & $4345(1)$ & 1972(1) & $594(1)$ & $23(1)$ \\
\hline$C(7)$ & $3110(1)$ & $4707(1)$ & $-716(1)$ & $24(1)$ \\
\hline$C(8)$ & 2695(1) & $6054(1)$ & $-620(1)$ & $26(1)$ \\
\hline $\mathrm{C}(9)$ & 2395(1) & 7164(1) & $-544(1)$ & $32(1)$ \\
\hline $\mathrm{C}(11)$ & 4999(1) & $2317(1)$ & 2713(1) & $22(1)$ \\
\hline$C(12)$ & $5685(1)$ & $3497(1)$ & $2766(1)$ & $24(1)$ \\
\hline$C(13)$ & $6056(1)$ & $4025(1)$ & $3480(1)$ & $24(1)$ \\
\hline$C(14)$ & $5780(1)$ & $3385(1)$ & $4159(1)$ & $22(1)$ \\
\hline$C(15)$ & $5125(1)$ & 2194(1) & $4105(1)$ & $22(1)$ \\
\hline$C(16)$ & 4733(1) & $1669(1)$ & 3393(1) & $23(1)$ \\
\hline $\mathrm{C}(17)$ & 6181(1) & $3977(1)$ & 4913(1) & $23(1)$ \\
\hline$C(18)$ & 5938(1) & $3228(1)$ & $5602(1)$ & $26(1)$ \\
\hline C(19) & $5756(2)$ & $2627(2)$ & $6165(1)$ & $34(1)$ \\
\hline $\mathrm{C}(21)$ & $4585(1)$ & $343(1)$ & 1946(1) & $23(1)$ \\
\hline$C(22)$ & $5687(1)$ & $-364(1)$ & 2188(1) & $24(1)$ \\
\hline C(23) & $5676(1)$ & $-1716(1)$ & $2139(1)$ & $27(1)$ \\
\hline$C(24)$ & $4561(1)$ & $-2367(1)$ & $1847(1)$ & $28(1)$ \\
\hline C(25) & $3468(1)$ & $-1657(1)$ & $1610(1)$ & $28(1)$ \\
\hline$C(26)$ & $3468(1)$ & $-302(1)$ & 1661(1) & $26(1)$ \\
\hline
\end{tabular}


Table S3. Bond lengths $[\AA]$ and angles $\left[^{\circ}\right]$ for $\mathbf{1}$.

$\begin{array}{ll}\mathrm{N}(1)-\mathrm{C}(1) & 1.4032(17) \\ \mathrm{N}(1)-\mathrm{C}(11) & 1.4094(17) \\ \mathrm{N}(1)-\mathrm{C}(21) & 1.4346(17) \\ \mathrm{O}(1)-\mathrm{C}(7) & 1.2235(16) \\ \mathrm{O}(2)-\mathrm{C}(17) & 1.2228(15) \\ \mathrm{C}(1)-\mathrm{C}(6) & 1.3993(19) \\ \mathrm{C}(1)-\mathrm{C}(2) & 1.4000(19) \\ \mathrm{C}(2)-\mathrm{C}(3) & 1.3745(19) \\ \mathrm{C}(3)-\mathrm{C}(4) & 1.3979(18) \\ \mathrm{C}(4)-\mathrm{C}(5) & 1.3927(18) \\ \mathrm{C}(4)-\mathrm{C}(7) & 1.4776(19) \\ \mathrm{C}(5)-\mathrm{C}(6) & 1.3740(19) \\ \mathrm{C}(7)-\mathrm{C}(8) & 1.4537(19) \\ \mathrm{C}(8)-\mathrm{C}(9) & 1.1848(19) \\ \mathrm{C}(11)-\mathrm{C}(16) & 1.3960(18) \\ \mathrm{C}(11)-\mathrm{C}(12) & 1.3978(18) \\ \mathrm{C}(12)-\mathrm{C}(13) & 1.3753(18) \\ \mathrm{C}(13)-\mathrm{C}(14) & 1.3952(18) \\ \mathrm{C}(14)-\mathrm{C}(15) & 1.3919(18) \\ \mathrm{C}(14)-\mathrm{C}(17) & 1.4681(18) \\ \mathrm{C}(15)-\mathrm{C}(16) & 1.3763(18) \\ \mathrm{C}(17)-\mathrm{C}(18) & 1.4564(19) \\ \mathrm{C}(18)-\mathrm{C}(19) & 1.180(2) \\ \mathrm{C}(21)-\mathrm{C}(22) & 1.3867(18) \\ \mathrm{C}(21)-\mathrm{C}(26) & 1.3877(18) \\ \mathrm{C}(22)-\mathrm{C}(23) & 1.3825(19) \\ \mathrm{C}(23)-\mathrm{C}(24) & 1.392(2) \\ \mathrm{C}(24)-\mathrm{C}(25) & 1.379(2) \\ \mathrm{C}(25)-\mathrm{C}(26) & 1.3856(19) \\ \mathrm{C}(1)-\mathrm{N}(1)-\mathrm{C}(11) & 122.62(11) \\ \mathrm{C}(1)-\mathrm{N}(1)-\mathrm{C}(21) & 119.80(11) \\ \mathrm{C}(11)-\mathrm{N}(1)-\mathrm{C}(21) & 117.57(11) \\ \mathrm{C}(6)-\mathrm{C}(1)-\mathrm{C}(2) & 118.32(12) \\ \mathrm{C}(6)-\mathrm{C}(1)-\mathrm{N}(1) & 119.90(12) \\ \mathrm{C}(2)-\mathrm{C}(1)-\mathrm{N}(1) & 121.76(12) \\ \mathrm{C}(3)-\mathrm{C}(2)-\mathrm{C}(1) & 120.57(13) \\ & \end{array}$




$\begin{array}{ll}\mathrm{C}(2)-\mathrm{C}(3)-\mathrm{C}(4) & 121.11(13) \\ \mathrm{C}(5)-\mathrm{C}(4)-\mathrm{C}(3) & 118.11(13) \\ \mathrm{C}(5)-\mathrm{C}(4)-\mathrm{C}(7) & 120.05(12) \\ \mathrm{C}(3)-\mathrm{C}(4)-\mathrm{C}(7) & 121.84(12) \\ \mathrm{C}(6)-\mathrm{C}(5)-\mathrm{C}(4) & 121.19(13) \\ \mathrm{C}(5)-\mathrm{C}(6)-\mathrm{C}(1) & 120.67(12) \\ \mathrm{O}(1)-\mathrm{C}(7)-\mathrm{C}(8) & 119.81(13) \\ \mathrm{O}(1)-\mathrm{C}(7)-\mathrm{C}(4) & 122.92(12) \\ \mathrm{C}(8)-\mathrm{C}(7)-\mathrm{C}(4) & 117.27(12) \\ \mathrm{C}(9)-\mathrm{C}(8)-\mathrm{C}(7) & 177.95(15) \\ \mathrm{C}(16)-\mathrm{C}(11)-\mathrm{C}(12) & 119.14(12) \\ \mathrm{C}(16)-\mathrm{C}(11)-\mathrm{N}(1) & 119.28(12) \\ \mathrm{C}(12)-\mathrm{C}(11)-\mathrm{N}(1) & 121.55(12) \\ \mathrm{C}(13)-\mathrm{C}(12)-\mathrm{C}(11) & 119.96(12) \\ \mathrm{C}(12)-\mathrm{C}(13)-\mathrm{C}(14) & 120.98(12) \\ \mathrm{C}(15)-\mathrm{C}(14)-\mathrm{C}(13) & 118.88(12) \\ \mathrm{C}(15)-\mathrm{C}(14)-\mathrm{C}(17) & 121.42(12) \\ \mathrm{C}(13)-\mathrm{C}(14)-\mathrm{C}(17) & 119.70(12) \\ \mathrm{C}(16)-\mathrm{C}(15)-\mathrm{C}(14) & 120.51(12) \\ \mathrm{C}(15)-\mathrm{C}(16)-\mathrm{C}(11) & 120.51(13) \\ \mathrm{O}(2)-\mathrm{C}(17)-\mathrm{C}(18) & 119.38(12) \\ \mathrm{O}(2)-\mathrm{C}(17)-\mathrm{C}(14) & 123.38(12) \\ \mathrm{C}(18)-\mathrm{C}(17)-\mathrm{C}(14) & 117.24(12) \\ \mathrm{C}(19)-\mathrm{C}(18)-\mathrm{C}(17) & 179.13(16) \\ \mathrm{C}(22)-\mathrm{C}(21)-\mathrm{C}(26) & 120.22(13) \\ \mathrm{C}(22)-\mathrm{C}(21)-\mathrm{N}(1) & 119.72(12) \\ \mathrm{C}(26)-\mathrm{C}(21)-\mathrm{N}(1) & 120.06(12) \\ \mathrm{C}(23)-\mathrm{C}(22)-\mathrm{C}(21) & 119.90(13) \\ \mathrm{C}(22)-\mathrm{C}(23)-\mathrm{C}(24) & 120.08(13) \\ \mathrm{C}(25)-\mathrm{C}(24)-\mathrm{C}(23) & 119.69(13) \\ \mathrm{C}(24)-\mathrm{C}(25)-\mathrm{C}(26) & 120.65(13) \\ \mathrm{C}(25)-\mathrm{C}(26)-\mathrm{C}(21) & 119.46(13) \\ & \end{array}$

Symmetry transformations used to generate equivalent atoms: 
Table S4. Anisotropic displacement parameters $\left(\AA^{2} \times 10^{3}\right)$ for $\mathbf{1}$. The anisotropic displacement factor exponent takes the form: $-2 \pi\left[h^{2} a^{* 2} U^{11}+\ldots+2 h k a^{*} b^{*} U^{12}\right]$

\begin{tabular}{|c|c|c|c|c|c|c|}
\hline & $\mathrm{U}^{11}$ & $\mathrm{U}^{22}$ & $\mathrm{U}^{33}$ & $\mathrm{U}^{23}$ & $\mathrm{U}^{13}$ & $\mathrm{U}^{12}$ \\
\hline $\mathrm{N}(1)$ & $31(1)$ & $22(1)$ & $19(1)$ & $-1(1)$ & $-2(1)$ & $0(1)$ \\
\hline $\mathrm{O}(1)$ & $39(1)$ & $31(1)$ & $21(1)$ & $2(1)$ & $2(1)$ & $6(1)$ \\
\hline $\mathrm{O}(2)$ & $36(1)$ & $26(1)$ & $29(1)$ & $-2(1)$ & $-6(1)$ & $-1(1)$ \\
\hline $\mathrm{C}(1)$ & $20(1)$ & $25(1)$ & $21(1)$ & $1(1)$ & $-1(1)$ & $-3(1)$ \\
\hline$C(2)$ & $28(1)$ & $26(1)$ & $20(1)$ & $-4(1)$ & $-1(1)$ & $-1(1)$ \\
\hline C(3) & $26(1)$ & $20(1)$ & $27(1)$ & $-3(1)$ & $-2(1)$ & $0(1)$ \\
\hline$C(4)$ & $20(1)$ & $23(1)$ & $23(1)$ & 1(1) & $0(1)$ & $-3(1)$ \\
\hline$C(5)$ & $24(1)$ & $25(1)$ & $21(1)$ & $-2(1)$ & $2(1)$ & $-4(1)$ \\
\hline$C(6)$ & $25(1)$ & $23(1)$ & $23(1)$ & $-1(1)$ & $3(1)$ & $0(1)$ \\
\hline$C(7)$ & $19(1)$ & $25(1)$ & $27(1)$ & $1(1)$ & $2(1)$ & $-2(1)$ \\
\hline$C(8)$ & $25(1)$ & $31(1)$ & $24(1)$ & $4(1)$ & $-1(1)$ & $-1(1)$ \\
\hline $\mathrm{C}(9)$ & $34(1)$ & $27(1)$ & $35(1)$ & $2(1)$ & $2(1)$ & $4(1)$ \\
\hline$C(11)$ & $20(1)$ & $24(1)$ & $21(1)$ & $-2(1)$ & $-2(1)$ & $3(1)$ \\
\hline$C(12)$ & $26(1)$ & $26(1)$ & $20(1)$ & $3(1)$ & $-1(1)$ & $-2(1)$ \\
\hline$C(13)$ & $25(1)$ & $21(1)$ & $26(1)$ & $-1(1)$ & $-1(1)$ & $-3(1)$ \\
\hline$C(14)$ & $20(1)$ & $24(1)$ & $21(1)$ & $-1(1)$ & $0(1)$ & $5(1)$ \\
\hline$C(15)$ & $23(1)$ & $23(1)$ & $21(1)$ & $1(1)$ & $3(1)$ & $2(1)$ \\
\hline$C(16)$ & $23(1)$ & $21(1)$ & $24(1)$ & $-1(1)$ & $2(1)$ & $1(1)$ \\
\hline$C(17)$ & $22(1)$ & $24(1)$ & $24(1)$ & $-2(1)$ & $-2(1)$ & $5(1)$ \\
\hline$C(18)$ & $23(1)$ & $31(1)$ & $23(1)$ & $-8(1)$ & $-3(1)$ & $1(1)$ \\
\hline$C(19)$ & $35(1)$ & $45(1)$ & $23(1)$ & $1(1)$ & $2(1)$ & $-2(1)$ \\
\hline$C(21)$ & $28(1)$ & $25(1)$ & $15(1)$ & $0(1)$ & 1(1) & $-2(1)$ \\
\hline$C(22)$ & $24(1)$ & $27(1)$ & $20(1)$ & $-4(1)$ & $2(1)$ & $-3(1)$ \\
\hline$C(23)$ & $29(1)$ & $29(1)$ & $23(1)$ & $-2(1)$ & $3(1)$ & $3(1)$ \\
\hline$C(24)$ & $39(1)$ & $23(1)$ & $23(1)$ & $-2(1)$ & $6(1)$ & $-5(1)$ \\
\hline$C(25)$ & $31(1)$ & $31(1)$ & $23(1)$ & $-1(1)$ & $0(1)$ & $-9(1)$ \\
\hline$C(26)$ & $26(1)$ & $29(1)$ & $21(1)$ & $2(1)$ & $-2(1)$ & $-1(1)$ \\
\hline
\end{tabular}


Table S5. Hydrogen coordinates $\left(\times 10^{4}\right)$ and isotropic displacement parameters $\left(\AA^{2} \times 10^{3}\right)$ for $\mathbf{1}$.

\begin{tabular}{|c|c|c|c|c|}
\hline & $\mathrm{X}$ & $\mathrm{y}$ & $\mathrm{z}$ & $\mathrm{U}(\mathrm{eq})$ \\
\hline $\mathrm{H}(2 \mathrm{~A})$ & 3602 & 4119 & 1883 & 30 \\
\hline $\mathrm{H}(3 \mathrm{~A})$ & 3037 & 5335 & 782 & 29 \\
\hline $\mathrm{H}(5 \mathrm{~A})$ & 4033 & 2305 & -554 & 28 \\
\hline $\mathrm{H}(6 \mathrm{~A})$ & 4690 & 1117 & 543 & 28 \\
\hline $\mathrm{H}(9)$ & 2155 & 8054 & -482 & 39 \\
\hline $\mathrm{H}(12 \mathrm{~A})$ & 5894 & 3936 & 2308 & 29 \\
\hline $\mathrm{H}(13 \mathrm{~A})$ & 6506 & 4836 & 3511 & 29 \\
\hline $\mathrm{H}(15 \mathrm{~A})$ & 4947 & 1739 & 4563 & 27 \\
\hline $\mathrm{H}(16 \mathrm{~A})$ & 4278 & 860 & 3363 & 27 \\
\hline H(19) & 5609 & 2143 & 6618 & 41 \\
\hline $\mathrm{H}(22 \mathrm{~A})$ & 6449 & 79 & 2387 & 29 \\
\hline $\mathrm{H}(23 \mathrm{~A})$ & 6431 & -2202 & 2304 & 32 \\
\hline $\mathrm{H}(24 \mathrm{~A})$ & 4553 & -3296 & 1811 & 34 \\
\hline$H(25 A)$ & 2707 & -2101 & 1410 & 34 \\
\hline $\mathrm{H}(26 \mathrm{~A})$ & 2708 & 181 & 1501 & 31 \\
\hline
\end{tabular}


Table S6. Torsion angles $\left[^{\circ}\right]$ for $\mathbf{1}$.

\begin{tabular}{|c|c|}
\hline $\mathrm{C}(11)-\mathrm{N}(1)-\mathrm{C}(1)-\mathrm{C}(6)$ & $154.54(12)$ \\
\hline $\mathrm{C}(21)-\mathrm{N}(1)-\mathrm{C}(1)-\mathrm{C}(6)$ & $-25.82(18)$ \\
\hline $\mathrm{C}(11)-\mathrm{N}(1)-\mathrm{C}(1)-\mathrm{C}(2)$ & $-26.87(19)$ \\
\hline $\mathrm{C}(21)-\mathrm{N}(1)-\mathrm{C}(1)-\mathrm{C}(2)$ & $152.77(12)$ \\
\hline $\mathrm{C}(6)-\mathrm{C}(1)-\mathrm{C}(2)-\mathrm{C}(3)$ & $-1.8(2)$ \\
\hline $\mathrm{N}(1)-\mathrm{C}(1)-\mathrm{C}(2)-\mathrm{C}(3)$ & $179.55(12)$ \\
\hline$C(1)-C(2)-C(3)-C(4)$ & $1.9(2)$ \\
\hline$C(2)-C(3)-C(4)-C(5)$ & $-0.37(19)$ \\
\hline$C(2)-C(3)-C(4)-C(7)$ & $-179.74(12)$ \\
\hline$C(3)-C(4)-C(5)-C(6)$ & $-1.19(19)$ \\
\hline$C(7)-C(4)-C(5)-C(6)$ & 178.19(12) \\
\hline$C(4)-C(5)-C(6)-C(1)$ & $1.2(2)$ \\
\hline$C(2)-C(1)-C(6)-C(5)$ & $0.30(19)$ \\
\hline$N(1)-C(1)-C(6)-C(5)$ & 178.94(12) \\
\hline $\mathrm{C}(5)-\mathrm{C}(4)-\mathrm{C}(7)-\mathrm{O}(1)$ & $3.4(2)$ \\
\hline $\mathrm{C}(3)-\mathrm{C}(4)-\mathrm{C}(7)-\mathrm{O}(1)$ & $-177.21(12)$ \\
\hline$C(5)-C(4)-C(7)-C(8)$ & $-175.77(11)$ \\
\hline$C(3)-C(4)-C(7)-C(8)$ & 3.59(19) \\
\hline $\mathrm{O}(1)-\mathrm{C}(7)-\mathrm{C}(8)-\mathrm{C}(9)$ & $-87(4)$ \\
\hline$C(4)-C(7)-C(8)-C(9)$ & $92(4)$ \\
\hline $\mathrm{C}(1)-\mathrm{N}(1)-\mathrm{C}(11)-\mathrm{C}(16)$ & $147.60(12)$ \\
\hline $\mathrm{C}(21)-\mathrm{N}(1)-\mathrm{C}(11)-\mathrm{C}(16)$ & $-32.05(17)$ \\
\hline $\mathrm{C}(1)-\mathrm{N}(1)-\mathrm{C}(11)-\mathrm{C}(12)$ & $-34.26(19)$ \\
\hline $\mathrm{C}(21)-\mathrm{N}(1)-\mathrm{C}(11)-\mathrm{C}(12)$ & $146.10(12)$ \\
\hline $\mathrm{C}(16)-\mathrm{C}(11)-\mathrm{C}(12)-\mathrm{C}(13)$ & $-1.80(19)$ \\
\hline N(1)-C(11)-C(12)-C(13) & $-179.95(12)$ \\
\hline $\mathrm{C}(11)-\mathrm{C}(12)-\mathrm{C}(13)-\mathrm{C}(14)$ & $1.2(2)$ \\
\hline $\mathrm{C}(12)-\mathrm{C}(13)-\mathrm{C}(14)-\mathrm{C}(15)$ & $0.33(19)$ \\
\hline $\mathrm{C}(12)-\mathrm{C}(13)-\mathrm{C}(14)-\mathrm{C}(17)$ & $-179.60(12)$ \\
\hline$C(13)-C(14)-C(15)-C(16)$ & $-1.31(19)$ \\
\hline $\mathrm{C}(17)-\mathrm{C}(14)-\mathrm{C}(15)-\mathrm{C}(16)$ & 178.62(12) \\
\hline $\mathrm{C}(14)-\mathrm{C}(15)-\mathrm{C}(16)-\mathrm{C}(11)$ & $0.74(19)$ \\
\hline $\mathrm{C}(12)-\mathrm{C}(11)-\mathrm{C}(16)-\mathrm{C}(15)$ & $0.83(19)$ \\
\hline $\mathrm{N}(1)-\mathrm{C}(11)-\mathrm{C}(16)-\mathrm{C}(15)$ & 179.02(12) \\
\hline $\mathrm{C}(15)-\mathrm{C}(14)-\mathrm{C}(17)-\mathrm{O}(2)$ & $-175.65(12)$ \\
\hline $\mathrm{C}(13)-\mathrm{C}(14)-\mathrm{C}(17)-\mathrm{O}(2)$ & $4.3(2)$ \\
\hline$C(15)-C(14)-C(17)-C(18)$ & 3.71(18) \\
\hline
\end{tabular}




$\begin{array}{lc}\mathrm{C}(13)-\mathrm{C}(14)-\mathrm{C}(17)-\mathrm{C}(18) & -176.36(11) \\ \mathrm{O}(2)-\mathrm{C}(17)-\mathrm{C}(18)-\mathrm{C}(19) & -37(11) \\ \mathrm{C}(14)-\mathrm{C}(17)-\mathrm{C}(18)-\mathrm{C}(19) & 144(11) \\ \mathrm{C}(1)-\mathrm{N}(1)-\mathrm{C}(21)-\mathrm{C}(22) & 126.68(13) \\ \mathrm{C}(11)-\mathrm{N}(1)-\mathrm{C}(21)-\mathrm{C}(22) & -53.67(16) \\ \mathrm{C}(1)-\mathrm{N}(1)-\mathrm{C}(21)-\mathrm{C}(26) & -53.29(17) \\ \mathrm{C}(11)-\mathrm{N}(1)-\mathrm{C}(21)-\mathrm{C}(26) & 126.37(13) \\ \mathrm{C}(26)-\mathrm{C}(21)-\mathrm{C}(22)-\mathrm{C}(23) & 0.52(19) \\ \mathrm{N}(1)-\mathrm{C}(21)-\mathrm{C}(22)-\mathrm{C}(23) & -179.44(11) \\ \mathrm{C}(21)-\mathrm{C}(22)-\mathrm{C}(23)-\mathrm{C}(24) & 0.00(19) \\ \mathrm{C}(22)-\mathrm{C}(23)-\mathrm{C}(24)-\mathrm{C}(25) & -0.2(2) \\ \mathrm{C}(23)-\mathrm{C}(24)-\mathrm{C}(25)-\mathrm{C}(26) & -0.1(2) \\ \mathrm{C}(24)-\mathrm{C}(25)-\mathrm{C}(26)-\mathrm{C}(21) & 0.6(2) \\ \mathrm{C}(22)-\mathrm{C}(21)-\mathrm{C}(26)-\mathrm{C}(25) & -0.83(19) \\ \mathrm{N}(1)-\mathrm{C}(21)-\mathrm{C}(26)-\mathrm{C}(25) & 179.13(11)\end{array}$

Symmetry transformations used to generate equivalent atoms: 


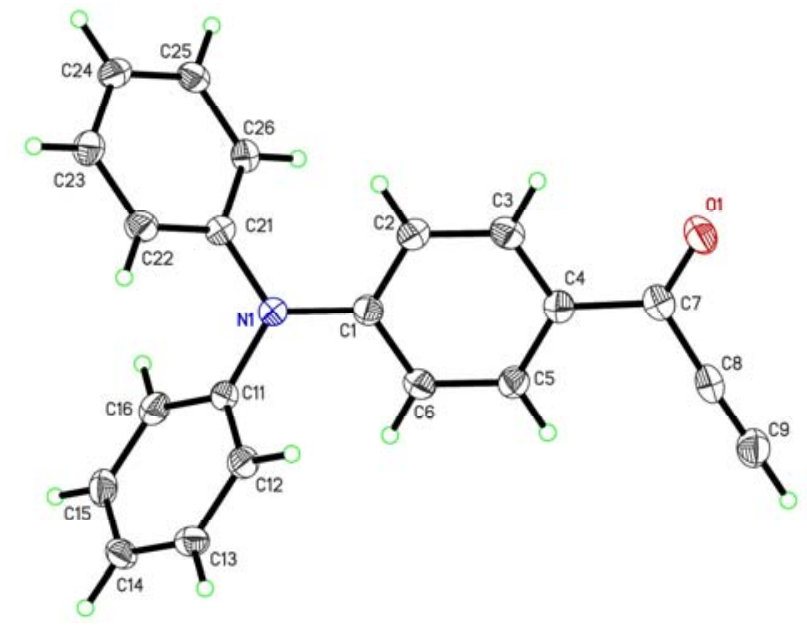

Figure S2. ORTEP drawing of $N$-(4-ethynylcarbonylphenylene)diphenylamine (4).

Table S7. Crystal data and structure refinement for 4.

\begin{tabular}{|c|c|c|}
\hline Empirical formula & \multicolumn{2}{|l|}{$\mathrm{C}_{21} \mathrm{H}_{15} \mathrm{NO}$} \\
\hline Formula weight & \multicolumn{2}{|l|}{297.34} \\
\hline Temperature & \multicolumn{2}{|l|}{$100(2) \mathrm{K}$} \\
\hline Wavelength & \multicolumn{2}{|l|}{$0.71073 \AA$} \\
\hline Crystal system & \multicolumn{2}{|l|}{ Monoclinic } \\
\hline Space group & \multicolumn{2}{|l|}{$\mathrm{P} 2(1) / \mathrm{c}$} \\
\hline \multirow[t]{3}{*}{ Unit cell dimensions } & $\mathrm{a}=15.3215(15) \AA$ & $\alpha=90^{\circ}$ \\
\hline & $\mathrm{b}=10.6382(11) \AA$ & $\beta=95.448(2)^{\circ}$ \\
\hline & $c=9.4347(9) \AA$ & $\gamma=90^{\circ}$ \\
\hline Volume & \multicolumn{2}{|l|}{$1530.8(3) \AA^{3}$} \\
\hline $\mathrm{Z}$ & \multicolumn{2}{|l|}{4} \\
\hline Density (calculated) & \multicolumn{2}{|l|}{$1.290 \mathrm{Mg} / \mathrm{m}^{3}$} \\
\hline Absorption coefficient & \multicolumn{2}{|l|}{$0.079 \mathrm{~mm}^{-1}$} \\
\hline $\mathrm{F}(000)$ & \multicolumn{2}{|l|}{624} \\
\hline Crystal size & \multicolumn{2}{|c|}{$0.40 \times 0.18 \times 0.15 \mathrm{~mm}^{3}$} \\
\hline Theta range for data collection & \multicolumn{2}{|c|}{2.33 to $27.00^{\circ}$. } \\
\hline Index ranges & \multicolumn{2}{|c|}{$-16<=\mathrm{h}<=19,-13<=\mathrm{k}<=13,-11<=\mathrm{l}<=12$} \\
\hline Reflections collected & \multicolumn{2}{|l|}{8952} \\
\hline Independent reflections & \multicolumn{2}{|c|}{$3197[\mathrm{R}(\mathrm{int})=0.0262]$} \\
\hline Completeness to theta $=25.00^{\circ}$ & \multicolumn{2}{|c|}{$96.5 \%$} \\
\hline Absorption correction & \multicolumn{2}{|c|}{ Semi-empirical from equivalents } \\
\hline Max. and min. transmission & \multicolumn{2}{|c|}{1.00 and 0.86} \\
\hline Refinement method & \multicolumn{2}{|c|}{ Full-matrix least-squares on $\mathrm{F}^{2}$} \\
\hline Data / restraints / parameters & \multicolumn{2}{|c|}{$3197 / 0$ / 208} \\
\hline Goodness-of-fit on $\mathrm{F}^{2}$ & \multicolumn{2}{|l|}{1.041} \\
\hline Final $\mathrm{R}$ indices [I>2sigma(I)] & \multicolumn{2}{|c|}{$\mathrm{R} 1=0.0357, \mathrm{wR} 2=0.0853$} \\
\hline $\mathrm{R}$ indices (all data) & \multicolumn{2}{|c|}{$\mathrm{R} 1=0.0519, \mathrm{wR} 2=0.0907$} \\
\hline Largest diff. peak and hole & \multicolumn{2}{|c|}{0.218 and -0.211 e. $\AA^{-3}$} \\
\hline
\end{tabular}


Table S8. Atomic coordinates $\left(\times 10^{4}\right)$ and equivalent isotropic displacement parameters $\left(\AA^{2} \times 10^{3}\right)$ for 4. $U(e q)$ is defined as one third of the trace of the orthogonalized $U^{\mathrm{ij}}$ tensor.

\begin{tabular}{lrrrr}
\hline & \multicolumn{3}{l}{ x } & $\mathrm{U}(\mathrm{eq})$ \\
\hline $\mathrm{O}(1)$ & $3509(1)$ & $5797(1)$ & $695(1)$ & $30(1)$ \\
$\mathrm{N}(1)$ & $7594(1)$ & $6330(1)$ & $2839(1)$ & $24(1)$ \\
$\mathrm{C}(1)$ & $6718(1)$ & $6415(1)$ & $2271(1)$ & $21(1)$ \\
$\mathrm{C}(2)$ & $6103(1)$ & $5501(1)$ & $2592(1)$ & $23(1)$ \\
$\mathrm{C}(3)$ & $5247(1)$ & $5581(1)$ & $2007(1)$ & $24(1)$ \\
$\mathrm{C}(4)$ & $4964(1)$ & $6571(1)$ & $1108(1)$ & $22(1)$ \\
$\mathrm{C}(5)$ & $5573(1)$ & $7487(1)$ & $797(1)$ & $23(1)$ \\
$\mathrm{C}(6)$ & $6438(1)$ & $7409(1)$ & $1364(1)$ & $24(1)$ \\
$\mathrm{C}(7)$ & $4042(1)$ & $6624(1)$ & $506(1)$ & $24(1)$ \\
$\mathrm{C}(8)$ & $3762(1)$ & $7727(1)$ & $-343(1)$ & $25(1)$ \\
$\mathrm{C}(9)$ & $3507(1)$ & $8608(1)$ & $-1035(1)$ & $30(1)$ \\
$\mathrm{C}(11)$ & $8127(1)$ & $7432(1)$ & $3037(1)$ & $21(1)$ \\
$\mathrm{C}(12)$ & $7821(1)$ & $8496(1)$ & $3681(1)$ & $23(1)$ \\
$\mathrm{C}(13)$ & $8365(1)$ & $9529(1)$ & $3934(1)$ & $24(1)$ \\
$\mathrm{C}(14)$ & $9215(1)$ & $9504(1)$ & $3557(1)$ & $25(1)$ \\
$\mathrm{C}(15)$ & $9520(1)$ & $8449(1)$ & $2901(1)$ & $26(1)$ \\
$\mathrm{C}(16)$ & $8977(1)$ & $7414(1)$ & $2631(1)$ & $25(1)$ \\
$\mathrm{C}(21)$ & $7968(1)$ & $5173(1)$ & $3366(1)$ & $21(1)$ \\
$\mathrm{C}(22)$ & $8491(1)$ & $5163(1)$ & $4660(1)$ & $25(1)$ \\
$\mathrm{C}(23)$ & $8874(1)$ & $4049(1)$ & $5165(1)$ & $25(1)$ \\
$\mathrm{C}(24)$ & $8721(1)$ & $2938(1)$ & $4420(1)$ & $24(1)$ \\
$\mathrm{C}(25)$ & $8195(1)$ & $2947(1)$ & $3138(1)$ & $22(1)$ \\
$\mathrm{C}(26)$ & $7830(1)$ & $4063(1)$ & $2596(1)$ & $21(1)$
\end{tabular}


Table S9. Bond lengths $[\AA]$ and angles $\left[^{\circ}\right]$ for 4 .

$\begin{array}{ll}\mathrm{O}(1)-\mathrm{C}(7) & 1.2245(13) \\ \mathrm{N}(1)-\mathrm{C}(1) & 1.4010(14) \\ \mathrm{N}(1)-\mathrm{C}(21) & 1.4268(13) \\ \mathrm{N}(1)-\mathrm{C}(11) & 1.4313(14) \\ \mathrm{C}(1)-\mathrm{C}(6) & 1.4008(15) \\ \mathrm{C}(1)-\mathrm{C}(2) & 1.4071(15) \\ \mathrm{C}(2)-\mathrm{C}(3) & 1.3758(16) \\ \mathrm{C}(3)-\mathrm{C}(4) & 1.3962(16) \\ \mathrm{C}(4)-\mathrm{C}(5) & 1.3984(15) \\ \mathrm{C}(4)-\mathrm{C}(7) & 1.4719(16) \\ \mathrm{C}(5)-\mathrm{C}(6) & 1.3847(15) \\ \mathrm{C}(7)-\mathrm{C}(8) & 1.4628(16) \\ \mathrm{C}(8)-\mathrm{C}(9) & 1.1867(17) \\ \mathrm{C}(11)-\mathrm{C}(12) & 1.3869(15) \\ \mathrm{C}(11)-\mathrm{C}(16) & 1.3914(15) \\ \mathrm{C}(12)-\mathrm{C}(13) & 1.3866(16) \\ \mathrm{C}(13)-\mathrm{C}(14) & 1.3833(16) \\ \mathrm{C}(14)-\mathrm{C}(15) & 1.3841(16) \\ \mathrm{C}(15)-\mathrm{C}(16) & 1.3894(16) \\ \mathrm{C}(21)-\mathrm{C}(26) & 1.3918(15) \\ \mathrm{C}(21)-\mathrm{C}(22) & 1.3953(16) \\ \mathrm{C}(22)-\mathrm{C}(23) & 1.3864(15) \\ \mathrm{C}(23)-\mathrm{C}(24) & 1.3841(15) \\ \mathrm{C}(24)-\mathrm{C}(25) & 1.3881(16) \\ \mathrm{C}(25)-\mathrm{C}(26) & 1.3893(15) \\ \mathrm{C}(1)-\mathrm{N}(1)-\mathrm{C}(21) & 121.84(9) \\ \mathrm{C}(1)-\mathrm{N}(1)-\mathrm{C}(11) & 120.75(9) \\ \mathrm{C}(21)-\mathrm{N}(1)-\mathrm{C}(11) & 117.16(9) \\ \mathrm{C}(6)-\mathrm{C}(1)-\mathrm{N}(1) & 120.59(10) \\ \mathrm{C}(6)-\mathrm{C}(1)-\mathrm{C}(2) & 118.77(11) \\ \mathrm{N}(1)-\mathrm{C}(1)-\mathrm{C}(2) & 120.64(10) \\ \mathrm{C}(3)-\mathrm{C}(2)-\mathrm{C}(1) & 120.16(11) \\ \mathrm{C}(2)-\mathrm{C}(3)-\mathrm{C}(4) & 121.34(10) \\ \mathrm{C}(3)-\mathrm{C}(4)-\mathrm{C}(5) & 118.54(11) \\ \mathrm{C}(3)-\mathrm{C}(4)-\mathrm{C}(7) & 119.62(10) \\ \mathrm{C}(5)-\mathrm{C}(4)-\mathrm{C}(7) & 121.83(10) \\ & \end{array}$




$\begin{array}{ll}\mathrm{C}(6)-\mathrm{C}(5)-\mathrm{C}(4) & 120.70(10) \\ \mathrm{C}(5)-\mathrm{C}(6)-\mathrm{C}(1) & 120.47(10) \\ \mathrm{O}(1)-\mathrm{C}(7)-\mathrm{C}(8) & 119.36(10) \\ \mathrm{O}(1)-\mathrm{C}(7)-\mathrm{C}(4) & 123.07(11) \\ \mathrm{C}(8)-\mathrm{C}(7)-\mathrm{C}(4) & 117.56(10) \\ \mathrm{C}(9)-\mathrm{C}(8)-\mathrm{C}(7) & 177.86(12) \\ \mathrm{C}(12)-\mathrm{C}(11)-\mathrm{C}(16) & 119.80(10) \\ \mathrm{C}(12)-\mathrm{C}(11)-\mathrm{N}(1) & 120.84(10) \\ \mathrm{C}(16)-\mathrm{C}(11)-\mathrm{N}(1) & 119.30(10) \\ \mathrm{C}(13)-\mathrm{C}(12)-\mathrm{C}(11) & 119.91(10) \\ \mathrm{C}(14)-\mathrm{C}(13)-\mathrm{C}(12) & 120.40(11) \\ \mathrm{C}(13)-\mathrm{C}(14)-\mathrm{C}(15) & 119.82(11) \\ \mathrm{C}(14)-\mathrm{C}(15)-\mathrm{C}(16) & 120.15(11) \\ \mathrm{C}(15)-\mathrm{C}(16)-\mathrm{C}(11) & 119.90(11) \\ \mathrm{C}(26)-\mathrm{C}(21)-\mathrm{C}(22) & 119.72(10) \\ \mathrm{C}(26)-\mathrm{C}(21)-\mathrm{N}(1) & 120.94(10) \\ \mathrm{C}(22)-\mathrm{C}(21)-\mathrm{N}(1) & 119.33(10) \\ \mathrm{C}(23)-\mathrm{C}(22)-\mathrm{C}(21) & 119.78(10) \\ \mathrm{C}(24)-\mathrm{C}(23)-\mathrm{C}(22) & 120.64(11) \\ \mathrm{C}(23)-\mathrm{C}(24)-\mathrm{C}(25) & 119.53(11) \\ \mathrm{C}(24)-\mathrm{C}(25)-\mathrm{C}(26) & 120.44(10) \\ \mathrm{C}(25)-\mathrm{C}(26)-\mathrm{C}(21) & 119.83(11) \\ & \end{array}$

Symmetry transformations used to generate equivalent atoms: 
Table S10. Anisotropic displacement parameters $\left(\AA^{2} \times 10^{3}\right)$ for 4 . The anisotropic displacement factor exponent takes the form: $-2 \pi^{2}\left[h^{2} a^{* 2} U^{11}+\ldots+2 h k a^{*} b^{*} U^{12}\right]$

\begin{tabular}{|c|c|c|c|c|c|c|}
\hline & $\mathrm{U}^{11}$ & $\mathrm{U}^{22}$ & $\mathrm{U}^{33}$ & $\mathrm{U}^{23}$ & $\mathrm{U}^{13}$ & $\mathrm{U}^{12}$ \\
\hline $\mathrm{O}(1)$ & $24(1)$ & $34(1)$ & $31(1)$ & $0(1)$ & $0(1)$ & $-7(1)$ \\
\hline $\mathrm{N}(1)$ & $20(1)$ & $18(1)$ & $32(1)$ & $1(1)$ & $-3(1)$ & $0(1)$ \\
\hline $\mathrm{C}(1)$ & $22(1)$ & $20(1)$ & $22(1)$ & $-4(1)$ & $0(1)$ & $1(1)$ \\
\hline$C(2)$ & $24(1)$ & $22(1)$ & $24(1)$ & $2(1)$ & $3(1)$ & $2(1)$ \\
\hline C(3) & $24(1)$ & $24(1)$ & $25(1)$ & $-3(1)$ & $6(1)$ & $-2(1)$ \\
\hline$C(4)$ & $21(1)$ & $24(1)$ & $20(1)$ & $-3(1)$ & $2(1)$ & 1(1) \\
\hline $\mathrm{C}(5)$ & $23(1)$ & $23(1)$ & $22(1)$ & $2(1)$ & $1(1)$ & $1(1)$ \\
\hline$C(6)$ & $22(1)$ & $22(1)$ & $27(1)$ & $1(1)$ & $3(1)$ & $-3(1)$ \\
\hline$C(7)$ & $23(1)$ & $29(1)$ & $20(1)$ & $-4(1)$ & $3(1)$ & $-2(1)$ \\
\hline $\mathrm{C}(8)$ & $18(1)$ & $33(1)$ & $23(1)$ & $-5(1)$ & $0(1)$ & $-3(1)$ \\
\hline $\mathrm{C}(9)$ & $26(1)$ & $34(1)$ & $28(1)$ & $2(1)$ & $-1(1)$ & $-1(1)$ \\
\hline $\mathrm{C}(11)$ & $20(1)$ & $20(1)$ & $21(1)$ & $3(1)$ & $-2(1)$ & $-1(1)$ \\
\hline$C(12)$ & $21(1)$ & $24(1)$ & $25(1)$ & $2(1)$ & $4(1)$ & $0(1)$ \\
\hline$C(13)$ & $29(1)$ & $20(1)$ & $24(1)$ & $-1(1)$ & $2(1)$ & $0(1)$ \\
\hline$C(14)$ & $25(1)$ & $24(1)$ & $24(1)$ & $5(1)$ & $-3(1)$ & $-7(1)$ \\
\hline$C(15)$ & $17(1)$ & $33(1)$ & $28(1)$ & $6(1)$ & $1(1)$ & $1(1)$ \\
\hline$C(16)$ & $23(1)$ & $24(1)$ & $26(1)$ & $0(1)$ & $1(1)$ & $6(1)$ \\
\hline$C(21)$ & $21(1)$ & $19(1)$ & $23(1)$ & $1(1)$ & $1(1)$ & $1(1)$ \\
\hline$C(22)$ & $28(1)$ & $21(1)$ & $24(1)$ & $-4(1)$ & $-1(1)$ & $-1(1)$ \\
\hline$C(23)$ & $27(1)$ & $27(1)$ & $21(1)$ & $2(1)$ & $-3(1)$ & $0(1)$ \\
\hline$C(24)$ & $26(1)$ & $20(1)$ & $26(1)$ & $3(1)$ & $3(1)$ & $2(1)$ \\
\hline$C(25)$ & $22(1)$ & $20(1)$ & $25(1)$ & $-3(1)$ & $4(1)$ & $-2(1)$ \\
\hline$C(26)$ & $19(1)$ & $24(1)$ & $21(1)$ & $-1(1)$ & $0(1)$ & $-1(1)$ \\
\hline
\end{tabular}


Table S11. Hydrogen coordinates $\left(\times 10^{4}\right)$ and isotropic displacement parameters $\left(\AA^{2} \times 10^{3}\right)$ for 4 .

\begin{tabular}{lrrrr}
\hline & \multicolumn{1}{c}{$\mathrm{x}$} & $\mathrm{y}$ & $\mathrm{z}$ & $\mathrm{U}(\mathrm{eq})$ \\
\hline $\mathrm{H}(2 \mathrm{~A})$ & 6279 & 4827 & 3215 & 28 \\
$\mathrm{H}(3 \mathrm{~A})$ & 4841 & 4949 & 2219 & 29 \\
$\mathrm{H}(5 \mathrm{~A})$ & 5390 & 8169 & 190 & 27 \\
$\mathrm{H}(6 \mathrm{~A})$ & 6845 & 8034 & 1137 & 28 \\
$\mathrm{H}(9)$ & 3303 & 9313 & -1589 & 36 \\
$\mathrm{H}(12 \mathrm{~A})$ & 7238 & 8516 & 3948 & 27 \\
$\mathrm{H}(13 \mathrm{~A})$ & 8152 & 10258 & 4370 & 29 \\
$\mathrm{H}(14 \mathrm{~A})$ & 9589 & 10208 & 3749 & 30 \\
$\mathrm{H}(15 \mathrm{~A})$ & 10103 & 8433 & 2634 & 31 \\
$\mathrm{H}(16 \mathrm{~A})$ & 9185 & 6695 & 2170 & 29 \\
$\mathrm{H}(22 \mathrm{~A})$ & 8584 & 5916 & 5193 & 29 \\
$\mathrm{H}(23 \mathrm{~A})$ & 9245 & 4049 & 6031 & 30 \\
$\mathrm{H}(24 \mathrm{~A})$ & 8974 & 2174 & 4783 & 28 \\
$\mathrm{H}(25 \mathrm{~A})$ & 8084 & 2184 & 2628 & 27 \\
$\mathrm{H}(26 \mathrm{~A})$ & 7488 & 4069 & 1702 & 25
\end{tabular}


Table S12. Torsion angles [ $\left.{ }^{\circ}\right]$ for 4.

$\begin{array}{lc}\mathrm{C}(21)-\mathrm{N}(1)-\mathrm{C}(1)-\mathrm{C}(6) & -159.40(10) \\ \mathrm{C}(11)-\mathrm{N}(1)-\mathrm{C}(1)-\mathrm{C}(6) & 26.67(15) \\ \mathrm{C}(21)-\mathrm{N}(1)-\mathrm{C}(1)-\mathrm{C}(2) & 20.20(16) \\ \mathrm{C}(11)-\mathrm{N}(1)-\mathrm{C}(1)-\mathrm{C}(2) & -153.74(10) \\ \mathrm{C}(6)-\mathrm{C}(1)-\mathrm{C}(2)-\mathrm{C}(3) & 0.84(16) \\ \mathrm{N}(1)-\mathrm{C}(1)-\mathrm{C}(2)-\mathrm{C}(3) & -178.76(10) \\ \mathrm{C}(1)-\mathrm{C}(2)-\mathrm{C}(3)-\mathrm{C}(4) & -1.13(17) \\ \mathrm{C}(2)-\mathrm{C}(3)-\mathrm{C}(4)-\mathrm{C}(5) & 0.57(16) \\ \mathrm{C}(2)-\mathrm{C}(3)-\mathrm{C}(4)-\mathrm{C}(7) & -179.84(10) \\ \mathrm{C}(3)-\mathrm{C}(4)-\mathrm{C}(5)-\mathrm{C}(6) & 0.26(16) \\ \mathrm{C}(7)-\mathrm{C}(4)-\mathrm{C}(5)-\mathrm{C}(6) & -179.32(10) \\ \mathrm{C}(4)-\mathrm{C}(5)-\mathrm{C}(6)-\mathrm{C}(1) & -0.52(17) \\ \mathrm{N}(1)-\mathrm{C}(1)-\mathrm{C}(6)-\mathrm{C}(5) & 179.57(10) \\ \mathrm{C}(2)-\mathrm{C}(1)-\mathrm{C}(6)-\mathrm{C}(5) & -0.03(16) \\ \mathrm{C}(3)-\mathrm{C}(4)-\mathrm{C}(7)-\mathrm{O}(1) & -3.96(16) \\ \mathrm{C}(5)-\mathrm{C}(4)-\mathrm{C}(7)-\mathrm{O}(1) & 175.61(11) \\ \mathrm{C}(3)-\mathrm{C}(4)-\mathrm{C}(7)-\mathrm{C}(8) & 175.53(10) \\ \mathrm{C}(5)-\mathrm{C}(4)-\mathrm{C}(7)-\mathrm{C}(8) & -4.89(15) \\ \mathrm{O}(1)-\mathrm{C}(7)-\mathrm{C}(8)-\mathrm{C}(9) & 4(3) \\ \mathrm{C}(4)-\mathrm{C}(7)-\mathrm{C}(8)-\mathrm{C}(9) & -175(3) \\ \mathrm{C}(1)-\mathrm{N}(1)-\mathrm{C}(11)-\mathrm{C}(12) & 47.36(15) \\ \mathrm{C}(21)-\mathrm{N}(1)-\mathrm{C}(11)-\mathrm{C}(12) & -126.85(11) \\ \mathrm{C}(1)-\mathrm{N}(1)-\mathrm{C}(11)-\mathrm{C}(16) & -135.36(11) \\ \mathrm{C}(21)-\mathrm{N}(1)-\mathrm{C}(11)-\mathrm{C}(16) & 50.43(14) \\ \mathrm{C}(16)-\mathrm{C}(11)-\mathrm{C}(12)-\mathrm{C}(13) & -0.84(17) \\ \mathrm{N}(1)-\mathrm{C}(11)-\mathrm{C}(12)-\mathrm{C}(13) & 176.43(10) \\ \mathrm{C}(11)-\mathrm{C}(12)-\mathrm{C}(13)-\mathrm{C}(14) & -0.44(17) \\ \mathrm{C}(12)-\mathrm{C}(13)-\mathrm{C}(14)-\mathrm{C}(15) & 1.13(17) \\ \mathrm{C}(13)-\mathrm{C}(14)-\mathrm{C}(15)-\mathrm{C}(16) & -0.54(17) \\ \mathrm{C}(14)-\mathrm{C}(15)-\mathrm{C}(16)-\mathrm{C}(11) & -0.73(17) \\ \mathrm{C}(12)-\mathrm{C}(11)-\mathrm{C}(16)-\mathrm{C}(15) & 1.42(17) \\ \mathrm{N}(1)-\mathrm{C}(11)-\mathrm{C}(16)-\mathrm{C}(15) & -175.89(10) \\ \mathrm{C}(1)-\mathrm{N}(1)-\mathrm{C}(21)-\mathrm{C}(26) & 45.82(15) \\ \mathrm{C}(11)-\mathrm{N}(1)-\mathrm{C}(21)-\mathrm{C}(26) & -140.04(11) \\ \mathrm{C}(1)-\mathrm{N}(1)-\mathrm{C}(21)-\mathrm{C}(22) & \\ \mathrm{C}(11)-\mathrm{N}(1)-\mathrm{C}(21)-\mathrm{C}(22) & \\ \mathrm{C}(26)-\mathrm{C}(21)-\mathrm{C}(22)-\mathrm{C}(23) & \\ & \end{array}$


$\mathrm{N}(1)-\mathrm{C}(21)-\mathrm{C}(22)-\mathrm{C}(23)$

$\mathrm{C}(21)-\mathrm{C}(22)-\mathrm{C}(23)-\mathrm{C}(24)$

$\mathrm{C}(22)-\mathrm{C}(23)-\mathrm{C}(24)-\mathrm{C}(25)$

$\mathrm{C}(23)-\mathrm{C}(24)-\mathrm{C}(25)-\mathrm{C}(26)$

$\mathrm{C}(24)-\mathrm{C}(25)-\mathrm{C}(26)-\mathrm{C}(21)$

$\mathrm{C}(22)-\mathrm{C}(21)-\mathrm{C}(26)-\mathrm{C}(25)$

$\mathrm{N}(1)-\mathrm{C}(21)-\mathrm{C}(26)-\mathrm{C}(25)$
$-178.44(10)$

$-2.05(17)$

1.54(17)

$0.60(16)$

$-2.22(16)$

1.69(17)

$-179.47(10)$

Symmetry transformations used to generate equivalent atoms: 